\title{
Circular RNA FOXO3 Suppresses Bladder Cancer Progression and Metastasis by Regulating MiR-9-5p/TGFBR2
}

This article was published in the following Dove Press journal: Cancer Management and Research

\section{Yongxiang $\mathrm{Li}^{1,2}$ Liang Qiao ${ }^{2}$ \\ Yuanwei Zang' \\ Wenjun $\mathrm{Ni}^{3}$ \\ Zhonghua $\mathrm{Xu}^{\mathrm{I}}$}

'Department of Urology, Qilu Hospital of Shandong University, Jinan 2500 I2, People's Republic of China; ${ }^{2}$ Department of Urology, Weifang People's Hospital, Weifang 261000, People's Republic of China; ${ }^{3}$ Department of Urology, Zhuhai People's Hospital (Zhuhai Hospital Affiliated with Jinan University), Zhuhai, People's Republic of China
Correspondence: Zhonghua Xu Department of Urology, Qilu Hospital of Shandong University, Jinan 2500I2, People's Republic of China

Tel +86-53I-8216670I

Fax +86-53I-86927544

Email bildsc@।63.com
Background: Increasing evidence indicates that the dysregulation of circular RNAs (circRNAs) plays important roles in tumor progressions.

Methods: In this study, we first analyzed circ-FOXO3 level in bladder cancers (BCs), and then $\mathrm{BC}$ cell lines were transfected with circ-FOXO3 expression vector, and cell proliferation, migration, and invasion abilities were analyzed. We also used bioinformatics tools to predict potential-binding miRNAs for circ-FOXO3, and luciferase reporter assay was used for the verification of binding miRNAs. For the further study, we analyzed potential downstream-binding mRNA for miRNA, and cell proliferation, migration and invasion abilities of it were also studied.

Results: We found that circ-FOXO3 was significantly down-regulated in bladder cancer (BC) tissues compared to normal bladder tissues. We also found that circ-FOXO3 overexpression inhibited cell proliferation, migration and invasion in $\mathrm{BC}$ cell lines. Moreover, we demonstrated that TGFBR2 was regulated by circ-FOXO3 through sponging miR-9-5p, the knockdown of TGFBR2 or the overexpression of miR-9-5p all related to the increased BC cell proliferation, migration, and invasion.

Discussion: In summary, our data showed that circ-FOXO3 was significantly downregulated in bladder cancers. circ-FOXO3 overexpression inhibits $\mathrm{BC}$ cell progression and metastasis. Furthermore, circ-FOXO3 regulates TGFBR2 expression through sponging miR9-5p in BC cell lines.

Keywords: bladder cancer, circ-FOXO3, miR-9-5p, TGFBR2

\section{Introduction}

Bladder cancer is a type of lethal cancer in the urogenital tract. Surgery, radiation therapy, and chemotherapy are the main therapies for $\mathrm{BC}$ patients. ${ }^{1,2}$ However, recurrence and progression are still related with different stages of patients. ${ }^{3}$ Therefore, the diagnosis of bladder cancer at early stages and the detailed research of the molecular mechanisms are essential for the diagnosis and treatment of the disease.

Non-coding RNAs (ncRNA) account for the majority of total RNAs in eukaryotic cells. Within all ncRNAs, circular RNAs are also a large part which have unique circular structures formed by binding both $3^{\prime}$ and $5^{\prime}$ ends of linear RNAs. ${ }^{4}$ Even though circular RNAs were reported for many years, their functions are still not well studied. A paper reported that some circular RNAs function as miRNA sponges and regulate their function. ${ }^{5}$ It has been reported that circRNA 
SRY can bind with miR-138 and function as a RNA sponge. ${ }^{4}$ Circ-7 can also work as the sponge to miR-7 to down-regulate its expression. ${ }^{6}$ miRNAs are well known to have multiple functions in regulating gene expressions and protein translations, thus circRNA might as well serve these functions by interacting with miRNAs. However, the full functions of circular RNAs are largely unknown. Foxo3 gene is one of the members of the forkhead family which are characterized by a unique forkhead domain within its gene. ${ }^{7}$ Foxo 3 related with apoptosis and cell death and it is also related to cancer progression. ${ }^{8}$ Besides, the expression of Foxo3 is reported to be up-regulated in acute leukemia. ${ }^{9}$ It was reported that circ-FOXO3 was related with breast cancer and played important roles in tumor genesis and progression, ${ }^{10,11}$ but in bladder cancer no relative study has been found.

In our study, we proved that circ-FOXO3 plays a critical role at cellular level which regulates cell growth and metastasis in bladder cancer, miR-9-5p was sponged by circFOXO3 and therefore regulates TGFBR2 to affect cell functions.

\section{Materials and Methods}

\section{Clinical Samples}

Tissue samples of bladder cancer and adjacent normal tissues were collected from patients diagnosed with bladder cancer $(n=49)$ from 2018 to 2019 from Qilu Hospital of Shandong University; the clinicopathological features are shown in Table 1. All procedures were approved by the Clinical Research Ethics Committee. All patients provided written informed consent and the study was conducted in accordance with the Declaration of Helsinki.

\section{Cell Culture and Transfection}

The bladder cancer cell lines EJ and T24 were purchased from cell bank of Chinese Academy of Sciences (Shanghai, China) and cultured in RPMI 1640 medium with 10\% fetal bovine serum (Invitrogen, Carlsbad, CA, USA), cells were cultured at $37^{\circ} \mathrm{C}$ in $5 \% \mathrm{CO}_{2}$. For cell transfection, circRNA-FOXO3 over expression vector (circRNA-FOXO3-OE) and its negative control, miR9-5p mimics (miR-9-5p-OE) and its negative control, siTGFBR2 vector and its negative control were all purchased from GenePharma (Shanghai, PR. China). For the transfection of target cells, when EJ and T24 cells
Table I Association Between Circ-FOXO3 Expression and Clinicopathological Features of Bladder Cancer Patients

\begin{tabular}{|c|c|c|c|c|}
\hline Variables & $\begin{array}{l}\text { Total } \\
(n=49)\end{array}$ & $\begin{array}{l}\text { High } \\
\text { Expression } \\
(n=22)\end{array}$ & $\begin{array}{l}\text { Low } \\
\text { Expression } \\
(n=27)\end{array}$ & $P$-value \\
\hline $\begin{array}{l}\text { Sex } \\
\qquad \text { Male } \\
\text { Female }\end{array}$ & $\begin{array}{l}36 \\
13\end{array}$ & $\begin{array}{l}14 \\
8\end{array}$ & $\begin{array}{l}22 \\
5\end{array}$ & $>0.05$ \\
\hline $\begin{array}{l}\text { Age } \\
\qquad 60 \\
\leq 60\end{array}$ & $\begin{array}{l}28 \\
21\end{array}$ & $\begin{array}{l}12 \\
10\end{array}$ & $\begin{array}{l}16 \\
11\end{array}$ & $>0.05$ \\
\hline $\begin{array}{l}\text { Tumor stage } \\
\text { Ta, Tis, } \\
\text { TI } \\
\text { T2-T4 }\end{array}$ & $\begin{array}{l}26 \\
23\end{array}$ & $\begin{array}{l}16 \\
6\end{array}$ & $\begin{array}{l}10 \\
17\end{array}$ & $<0.05$ \\
\hline $\begin{array}{l}\text { Lymph node } \\
\text { metastasis } \\
\text { Present } \\
\text { Absent }\end{array}$ & $\begin{array}{l}32 \\
17\end{array}$ & $\begin{array}{l}10 \\
12\end{array}$ & $\begin{array}{l}22 \\
5\end{array}$ & $<0.01$ \\
\hline $\begin{array}{l}\text { Distant } \\
\text { metastasis } \\
\text { Present } \\
\text { Absent }\end{array}$ & $\begin{array}{l}11 \\
38\end{array}$ & $\begin{array}{l}2 \\
20\end{array}$ & $\begin{array}{l}8 \\
18\end{array}$ & $>0.05$ \\
\hline
\end{tabular}

cultured to $80 \%$ confluence, Lipofectamine 2000 (Invitrogen) were used for transfection according manufacturer's instructions.

\section{Real-Time PCR}

Total RNAs were extracted from cells by using Trizol reagent (Invitrogen, USA) and quantitative Real-Time PCR (qRTPCR) were then performed according to the manufacturer's protocol. The expression of miR-9-5p were tested by using TaqMan microRNA Assays kit (Applied Biosystems, USA), U6 and GAPDH were used as internal controls, and $2^{-\Delta \Delta \mathrm{ct}}$ was used to evaluate relative expressions. All primers used were shown as follows: circRNA-FOXO3 forward: $5^{\prime}$-GT GGGGAACTTCACTGGTGCTAAG-3', reverse: 5'-GGGT TGATGATCC ACCAAGAGCTCTT-3'; miR-9-5p forward: 5'-GTGCAGGGTCCGAGGT-3', reverse: 5'-GCGCTCTTT GGTTATCTAGC-3'; TGFBR2 forward: 5'-GACCAGAA ATTCCCAGCTTCT-3', reverse: 5'- CAACGTCTCACACA CCATCTG-3'; GAPDH forward: 5' -ACTTTGGTATCGT GGAAGGACT-3', reverse:5' -GTAGAGGCAGGGATGAT GTTCT-3'; U6 forward: 5'-AGCCCGCACTCAGAACATC -3 ', reverse: 5'-GCCACCAAGACAATCATCC-3'. 


\section{Western Blot}

For Western blot assay, cultured EJ and T24 cells were lysed in RIPA lysis buffer (Beyotime, China). For each lane, proteins were loaded and $10 \%$ SDS denatured polyacrylamide gel electrophoresis were performed, then protein were transferred onto a nitrocellulose membrane. After blocking with 5\% BSA with TBST sodium, membrane was then incubated with primary antibody overnight at $4{ }^{\circ} \mathrm{C}$. On the other day, after washing with TBST, blots were incubated with secondary antibody at room temperature for 1 hour. After incubation, membrane was washed again and visualized by using ECL-Plus reagent (Millipore, USA).

\section{Luciferase Reporter Assay}

For the luciferase reporter assay, we seeded both EJ and T24 cells into 24 -well plates at $1 \times 10^{5}$ per well. Cells were cotransfected with both miR-9-5p mimics (or control vector) and wild-type (or mutated) TGFBR2 (or circRNA-FOXO3) plasmids. Lipofectamine 2000 reagent (Invitrogen) were used for transfection. Forty-eight hours after transfection, different luciferase activities were assessed using dualluciferase reporter kit (Promega, USA). Relative firefly luciferase levels were normalized to renilla luciferase levels.

\section{Cell Proliferation Assay}

Cell Counting Kit-8 (7 sea biotech, Shanghai, China) were used for analyzing cell proliferation. Cells were first seeded into 96-well plates at $1 \times 104$ per well and incubated $5 \% \mathrm{CO}_{2}$ at $37^{\circ} \mathrm{C}$ until cell confluence at $70 \%$. Forty-eight hours after transfection, cells were incubated for $24,48,72$, and 96 hours. Each well then had $10 \mu 1$ of CCK8 solution added. Absorbance at $450 \mathrm{~nm}$ was measured by using a SUNRISE Microplate Reader (Tecan, Switzerland).

\section{Cell Migration and Invasion Assay}

Twenty-four-well transwell plates (Corning, USA) were used for measuring cell migratory and invasive ability. For migration assays, $2 \times 104$ cells were added into the top chamber, chamber inserts were coated with $200 \mathrm{mg} / \mathrm{mL}$ of matrigel and dried overnight. Twenty-four hours after incubation at $37^{\circ} \mathrm{C}$, top chambers were wiped with cotton wool to remove noninvasive cells. Cells on the underside of the membrane were then fixed with $100 \%$ methanol for $10 \mathrm{~min}$ utes, air-dried and stained with $0.1 \%$ crystal violet. Cells were then visualized with microscope (Leica Inc.,
Germany) and quantified by counting the number of cells in three random fields.

\section{Statistical Analysis}

All results were presented as mean $\pm \mathrm{SD}$ and analyzed by using SPSS 19.0, (SPSS Inc., USA). For the comparison of different groups, Student's $t$-test and one-way ANOVA were used and $P<0.05$ was considered to be significantly different.

\section{Results \\ Down-Regulated Expression of Circ-FOXO3 in Bladder Cancer}

To explore whether circRNAs play important roles in tumor progression of bladder cancer, we analyzed datasets of circRNA sequencing of bladder cancers. We found out a series of down-regulated expression of circRNAs in bladder cancer tissues compared to normal tissues according to dataset (GSE97239) (Figure 1A). Among all these circRNAs we found circ-FOXO3 to be one of the most down-regulated circRNAs and we decided to further investigate it. To confirm this result, we performed PCR to validate the expression level of circ-FOXO3 in bladder cancers and adjacent normal tissues, results indicated that circ-FOXO3 were significantly down-regulated in $\mathrm{BCs}$ (Figure 1B). We then analyzed the expression level of circFOXO3 level within most common BC cell lines, both EJ and T24 were chosen to be ideal cell lines for further investigations (Figure 1C). To find out the correlation between circ-FOXO3 expression levels and $\mathrm{BC}$ progression, we analyzed the correlation between circ-FOXO3 expression and clinical features in BC patients. KaplanMeier analysis showed that the low expression level of circ-FOXO3 correlated with poor survival rates in $\mathrm{BC}$ patients (Figure 1D). All these data indicated that low circFOXO3 expression related with $\mathrm{BC}$ progression.

\section{The Overexpression of Circ-FOXO3}

\section{Suppressed Cell Growth, Migration, and Invasion}

To investigate the effects of circ-FOXO3 on $\mathrm{BC}$ cells, we transfected both EJ and T24 cells with circ-FOXO3 expressing vector. After transfection, circ-FOXO3 expression was markedly upregulated in EJ and T24 cells (Figure 2A). Then we performed CCK8 assays, data showed that circ-FOXO3 overexpression suppressed the proliferation in EJ and T24 cells (Figure 2B and C). Transwell assays also indicated that 


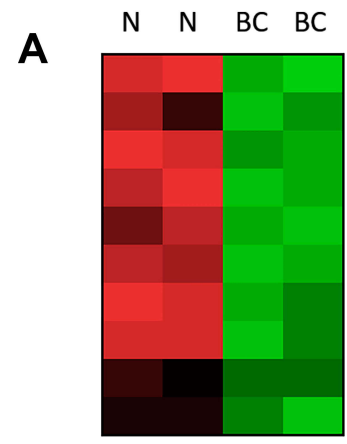

Circ-FOXO3

Circ-0083713

Circ-0001283

Circ-GBAS

Circ-ptk2

Circ-0067934

Circ-C3P1

Circ-ZEB1

Circ-102231

Circ-001678

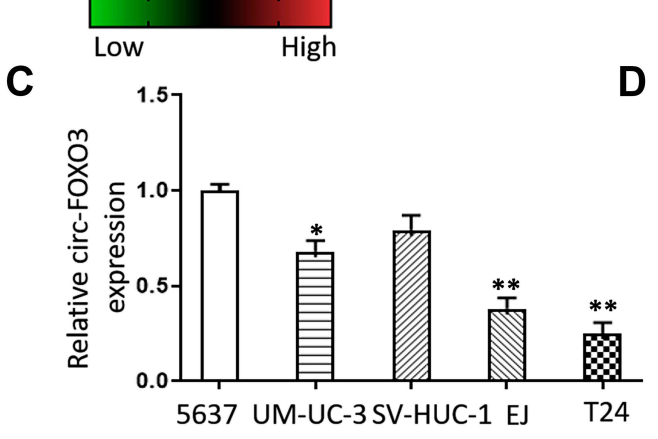

B
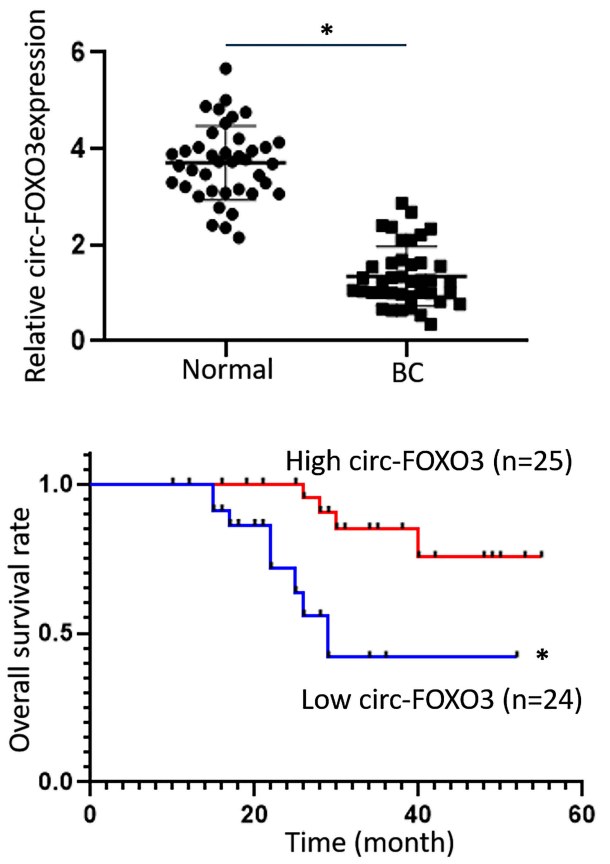

Figure I The expression of circ-FOXO3 was down-regulated in bladder cancer tissues and cell lines. (A) The down-regulated expression of circRNAs in bladder cancer tissues compared to normal tissues according to dataset (GSE97239). (B) The expression of circ-FOXO3 in bladder cancer tissues and adjacent normal tissues ( $\mathrm{n}=49$ ). (C) The expressions of circ-FOXO3 in bladder cancer cell lines. (D) Kaplan-Meier survival analysis of circ-FOXO3 expressions in bladder cancer patients. ${ }^{*}<<0.05$ compared with the control group, $* * P<0.01$ compared with the control group.

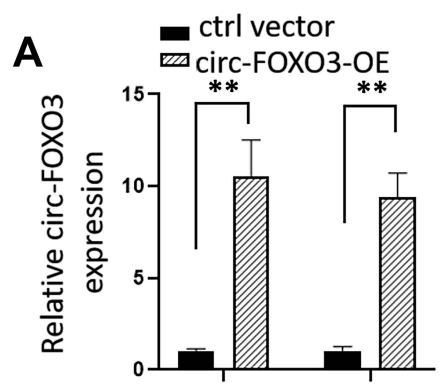

D

EJ migration

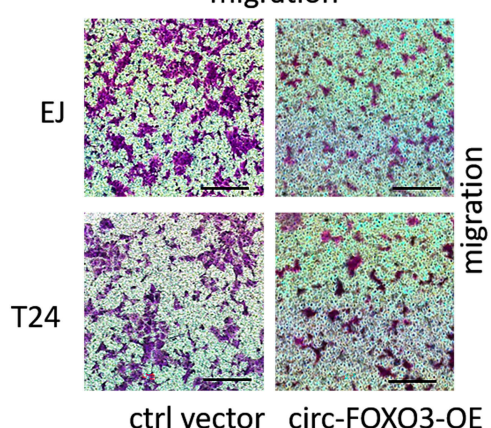

ctrl vector circ-FOXO3-OE
B

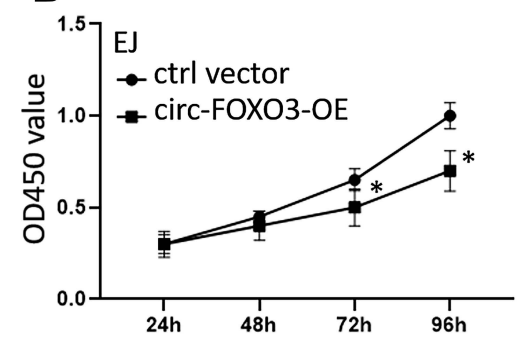

E

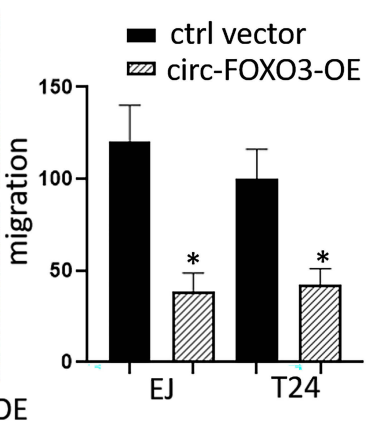

$\mathbf{F}$

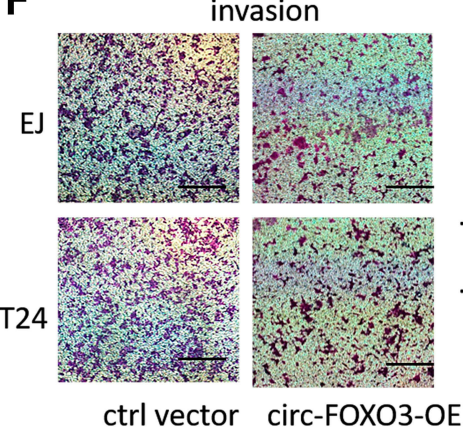

C

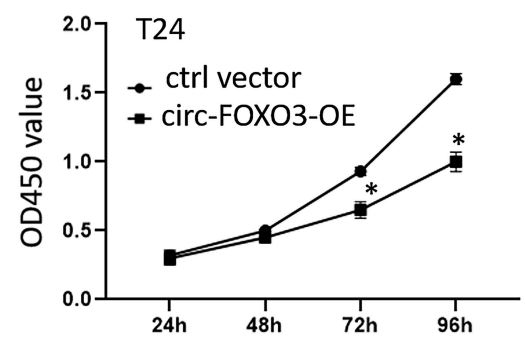

G

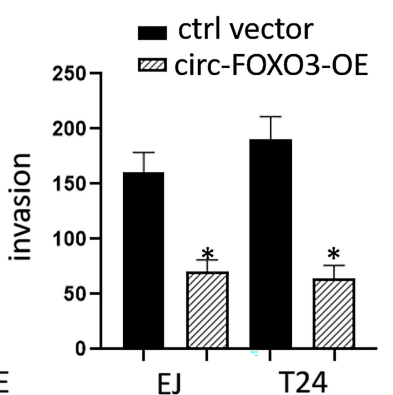

Figure 2 Overexpression of circ-FOXO3 attenuated cell growth, migration, and invasion of bladder cancer cells. (A) The expression levels of circ-FOXO3 in bladder cancer cell lines EJ and T24 after the transfection of circ-FOXO3 expression vectors. (B and C) Cell proliferation levels of both EJ and T24 after the transfection of circ-FOXO3 expression vectors which is measured by CCK8 assay. ( $D$ and $\mathbf{E}$ ) Cell migration assay of both EJ and T24 after the transfection of circ-FOXO3 expression vectors (scale bar: $200 \mu \mathrm{m})$. (F and $\mathbf{G})$ Cell invasion assay of both EJ and T24 after the transfection of circ-FOXO3 expression vectors (scale bar: $200 \mu \mathrm{m})$. $* P<0.05$ compared with the control group, $* * P<0.01$ compared with the control group. 
circ-FOXO3 overexpression remarkably suppressed cell migration and invasion abilities in both EJ and T24 cells (Figure 2D-G). These data proved the tumor suppression ability of circ-FOXO3 in BC cells.

\section{Circ-FOXO3 Regulated MiR-9-5p/ TGFBR2 Pathway in Bladder Cancer Cells}

To further explore the mechanism of circ-FOXO3 in $\mathrm{BC}$ cells, we use bioinformatics tools (miRanda and TargetScan) to predict the potential binding miRNAs of circ-FOXO3, after researching of all predicted results, we found miR-9-5p is a potential candidate for further investigation. We then find the potential binding sites on miR-9-5p and the downstream gene TGFBR2 which can be regulated by miR-9-5p
(Figure 3A and B). We used circ-FOXO3 expressing vector to transfect EJ and T24 cells, results showed significantly up-regulated expression of circ-FOXO3 (Figure 3C). Then we used the miR-9-5p expression vector to co-transfect BC cells with either circ-FOXO3 wild type or mutant vectors, after transfection, luciferase level of cells in each group was measured. Results showed that BC cells with both circFOXO3 WT and miR-9-5p expression vector had a lower luciferase level than cells with both circ-FOXO3 mutant and miR-9-5p expression vector (Figure 3D). To further investigate whether miR-9-5p has an impact on TGFBR2, we used miR-9-5p expression vector to co-transfect BC cells with either TGFBR2 wild type or mutant vectors, after transfection, luciferase level of cells in each group was measured.

A

Circ-FOXO3 WT 5'-AACCUGAUACCUGUUACCAAAGC-3' miR-9-5p 3'-AGUAUGUCGAUCUAUUGGUUUCU-5' Circ-FOXO3 mutant 5 '-AACCUGAUACCUGUUUGGUUUCC-3'

B

TGFBR2 3'UTR WT 5'-GAGGCUGCCCCUCUCACCAAAGA-3' miR-9-5p 3'-AGUAUGUCGAUCUAUUGGUUUCU-5' TGFBR2 3'UTR mutant 5'-GAGGCUGCCCCUCUCUGGUUUCA-3'

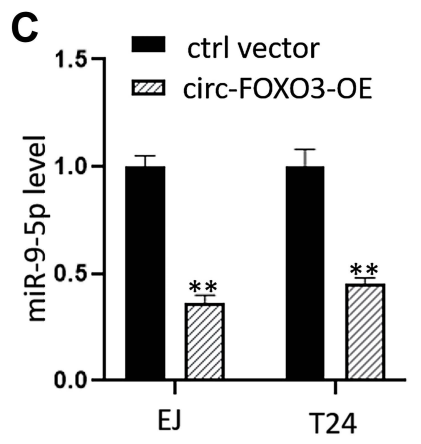

D

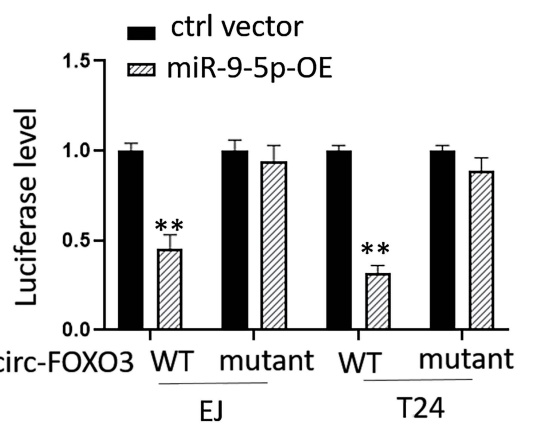

$\mathbf{F}$

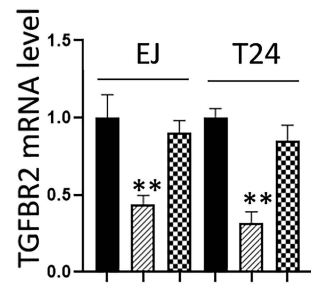

circ-FOXO3-OE ++++++

SiTGFBR2 - ++-++

TGFBR2-OE - - + - - +
E
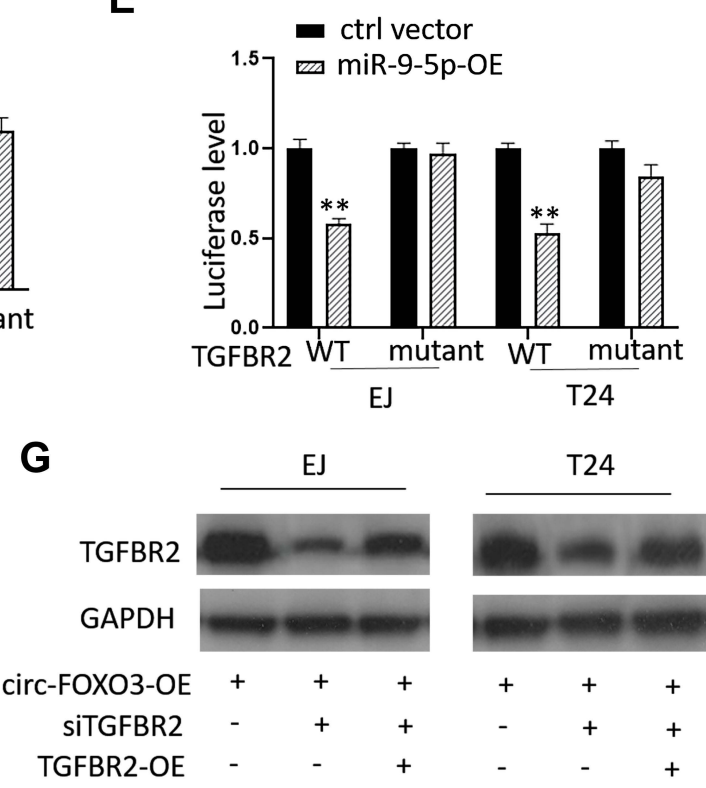

Figure 3 Circ-FOXO3 regulated TGFBR2 expression by targeting miR-9-5p. (A and B) The schematic maps of putative binding targets on both miR-9-5p and TGFBR2. (C) Relative miR-9-5p levels in EJ and T24 cells after the transfection of circ-FOXO3 expression vectors. (D) Relative luciferase levels in EJ and T24 cells after the co-transfection of miR-9-5p expression vector and circ-FOXO3 wild type or mutant vector. (E) Relative luciferase levels in EJ and T24 cells after the co-transfection of miR-9-5p expression vector and TGFBR2 wild type or mutant vector. (F) Relative TGFBR2 mRNA levels in EJ and T24 cells after the transfection of circ-FOXO3 expression vector, circ-FOXO3 expression vector with TGFBR2 siRNA or circ-FOXO3 expression vector, TGFBR2 siRNA and TGFBR2 expression vector. (G) Relative TGFBR2 protein levels in EJ and T24 cells after the transfection of circ-FOXO3 expression vector, circ-FOXO3 expression vector with TGFBR2 siRNA or circ-FOXO3 expression vector, TGFBR2 siRNA and TGFBR2 expression vector. ${ }^{* * P}<0.01$. 
Results showed that BC cells with both TGFBR2 WT and miR-9-5p expression vector had lower luciferase level than cells with both TGFBR2 mutant and miR-9-5p expression vector (Figure 3E). We then measured both mRNA and protein level of TGFBR2 after circ-FOXO3 expression vector transfection only or co-transfection of circ-FOXO3 expression vector and TGFBR2 siRNA, results showed significantly down-regulated TGFBR2 expression in both mRNA and protein level (Figure $3 \mathrm{~F}$ and $\mathrm{G}$ ). We then checked TGFBR2 level by transfecting cells with RGFBR2 expression vector after the co-transfection of circFOXO3 expression vector and TGFBR2 siRNA, results showed up-regulated level of TGFBR2 compared with siRNA group (Figure $3 \mathrm{~F}$ and $\mathrm{G}$ ). Taken together, all these data demonstrated circ-FOXO3 overexpression inhibits miR-9-5p expression and the down-regulated
miR-9-5p promotes TGFBR2 transcription and translation expression in $\mathrm{BC}$ cells.

\section{Circ-FOXO3 Regulated Cell Proliferation, Migration and Invasion by Affecting TGFBR2 Expression}

To further investigate the effects of TGFBR2 in BC cells, we first tested TGFBR2 level in BC tissues and adjacent normal tissues. Results showed a significantly decreased mRNA expression level of TGFBR2 in BC tissues compared to normal tissues (Figure 4A). We then transfected both EJ and T24 cells with circ-FOXO3 expression vector or both circ-FOXO3 expression vector and TGFBR2 siRNA. CCK8 assays showed that circFOXO3 overexpression suppressed the proliferation in EJ and T24 cells and TGFBR2 siRNA attenuated this

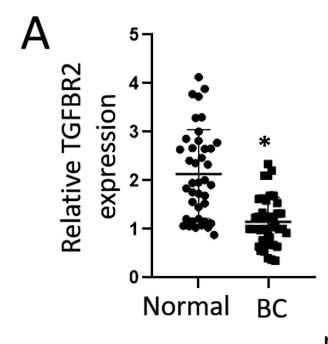

D

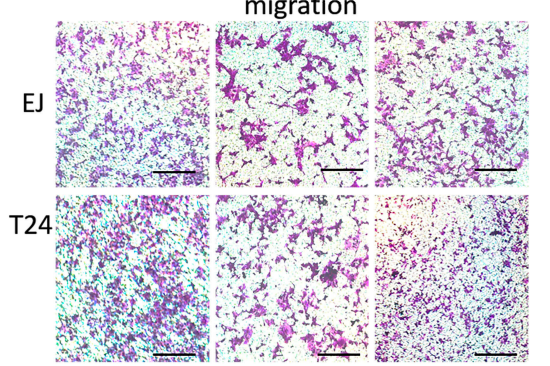

ctrl vector circ-FOXO3-OE circ-FOXO3-OE + siTGFBR2

$\mathrm{F}$

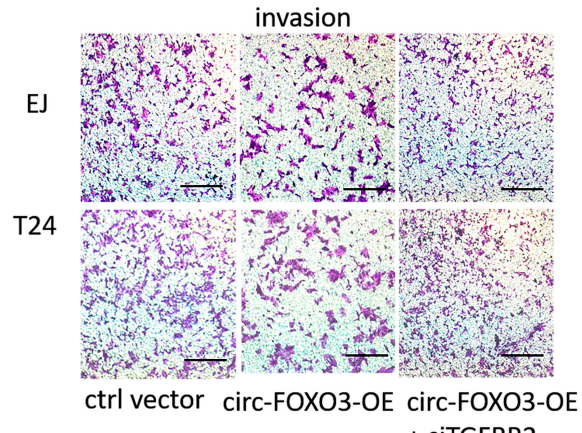

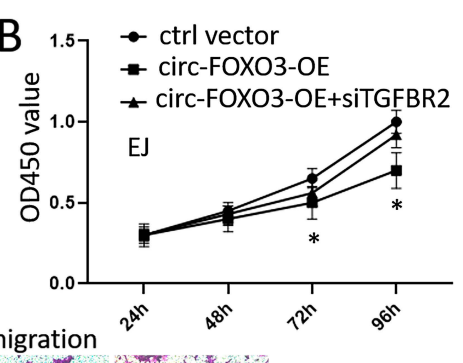

${ }^{2.0} \rightarrow$ ctrl vector

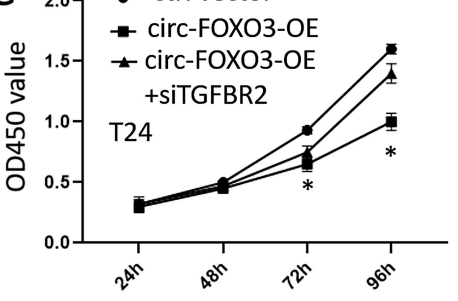

E

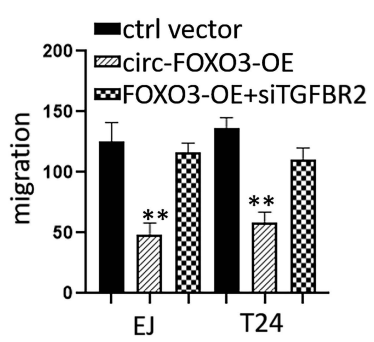

G

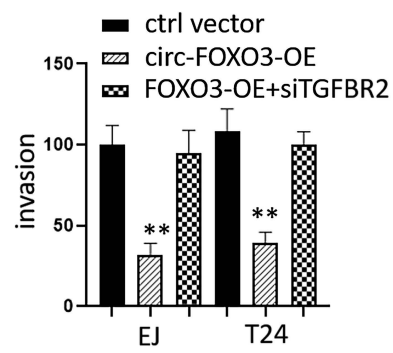

Figure 4 Overexpression of circ-FOXO3 attenuated cell growth, migration, and invasion of bladder cancer cells by regulating TGFBR2. (A) The different mRNA expression levels of TGFBR2 in bladder cancer tissues and adjacent normal tissues $(n=49)$. (B and C) Cell proliferation levels of both EJ and T24 after the transfection of circ-FOXO3 expression vectors or both circ-FOXO3 expression vectors and TGFBR2 siRNA measured by CCK8 assay. (D and E) Cell migration assay of both EJ and T24 after the transfection of circ-FOXO3 expression vectors or both circ-FOXO3 expression vectors and TGFBR2 siRNA (scale bar: $200 \mu \mathrm{m})$. (F and $\mathbf{G})$ Cell invasion assay of both EJ and T24 after the transfection of circ-FOXO3 expression vectors both circ-FOXO3 expression vectors and TGFBR2 siRNA (scale bar: $200 \mu \mathrm{m}$ ). $* P<0.05$ compared with the control group, $* * P<0.01$ compared with the control group. 
effect (Figure 4B and C). Trans-well assays indicated that circ-FOXO3 overexpression remarkably suppressed cell migration and invasion abilities in both EJ and T24 cells, cells transfected with both circ-FOXO3 expression vector and TGFBR2 siRNA had greater migration and invasion abilities compared with the circ-FOXO3 expression vector transfected only group (Figure 4D-G). These data indicated the tumor suppression ability of circFOXO3 in BC cells was achieved by inhibiting miR$9-5 p$ and promoting TGFBR2 expression.

\section{Discussion}

Recently the function of a special circRNA which named circ-FOXO3 was studied by Burton Yang, who found that circ-FOXO3 was down-regulated in a series of cancers. ${ }^{12}$ In our study, we also found the lower level of circ-FOXO3 in $\mathrm{BC}$ tissues compared with adjacent normal tissues. Results also indicated that the expression of circ-FOXO3 suppressed cell proliferation and invasion in $\mathrm{BC}$. To find out the correlation between circ-FOXO3 expression levels and $\mathrm{BC}$ progression, we analyzed the correlation between circ-FOXO3 expression and clinical features in $\mathrm{BC}$ patients. Kaplan-Meier analysis showed that low expression level of circ-FOXO3 correlated with poor survival rates in $\mathrm{BC}$ patients. All these data indicated that low circFOXO3 expression related with $\mathrm{BC}$ progression.

MicroRNAs (miRNAs) are a class of non-coding RNAs which related with multiple functions in cells. ${ }^{13}$ It has been reported that miRNAs are closely related to the translation of mRNAs and also have impacts on cancer migration and invasion. ${ }^{14}$ In this study, we use bioinformatics tools to predict the potential binding miRNAs of circ-FOXO3, after researching of all predicted results, we found miR$9-5 p$ is a potential candidate for further investigation. We used circ-FOXO3 expressing vector to transfect EJ and T24 cells, results showed significantly up-regulated expression of circ-FOXO3. Then we used this expressing vector to transfect $\mathrm{BC}$ cells with either circ-FOXO3 wild type/mutant vectors or TGFBR2 wild type/mutant vectors, after cotransfection we analyzed luciferase level of $\mathrm{BC}$ cells. Results showed the significantly lower luciferase level in circ-FOXO3 WT vector and TGFBR2 WT vector groups, but in mutant vectors groups, this phenomenon were vanished. We then measured both mRNA and protein level of TGFBR2 after circ-FOXO3 over expressing vector transfection only or co-transfection of circ-FOXO3 expression vector and TGFBR2 siRNA, results showed significantly down-regulated TGFBR2 expression in both mRNA and protein level. Taken together, all these data demonstrated circ-FOXO3 regulates miR-9-5p expression, which regulates TGFBR2 transcription and translation expression in $\mathrm{BC}$ cells.

Foxo3 gene which encodes both linear and circular Foxo3 has been reported to be associated with cancer progression and development, it is also related with Akt and Phosphatase and tension homolog (PTEN) signaling pathways. ${ }^{15}$ To investigate the effects of circ-FOXO3 on $\mathrm{BC}$ cells, we transfected both EJ and T24 cells with circFOXO3 expressing vector. After transfection, circ-FOXO3 expression was markedly upregulated in EJ and T24 cells. CCK8 assays showed that circ-FOXO3 overexpression suppressed the proliferation in EJ and T24 cells. Trans-well assays also indicated that circ-FOXO3 overexpression remarkably suppressed cell migration and invasion abilities. These data proved the tumor suppression ability of circFOXO3 in BC cells. To further investigate the effects of TGFBR2 in BC cells, we first tested TGFBR2 level in BC tissues and adjacent normal tissues. Results showed a significantly decreased mRNA expression level of TGFBR2 in BC tissues compared to normal tissues. We then transfected both EJ and T24 cells with circ-FOXO3 expression vector or both circ-FOXO3 expression vector and TGFBR2 siRNAs. CCK8 assays showed that circFOXO3 overexpression suppressed the proliferation in EJ and T24 cells and TGFBR2 siRNA blocked this effect. Trans-well assays indicated that circ-FOXO3 overexpression remarkably suppressed cell migration and invasion abilities in both EJ and T24 cells, cells transfected with both circ-FOXO3 expression vector and TGFBR2 siRNA had greater migration and invasion abilities compared with circ-FOXO3 expression vector transfection only group. These data indicated the tumor suppression ability of circFOXO3 in BC cells was achieved by inhibiting miR-9-5p and promoting TGFBR2 expression.

In summary, in this study our data showed that the upregulated circ-FOXO3 could inhibit cell proliferation and metastasis in bladder cancers. Furthermore, circ-FOXO3 promotes TGFBR2 expression through inhibiting miR$9-5 \mathrm{p}$ expression in $\mathrm{BC}$ cells. Therefore, circ-FOXO3 as a tumor suppressor can serve as the prognostic biomarker and potential therapeutic target in $\mathrm{BCs}$.

\section{Funding}

This work was supported by the fund of Qilu Hospital of Shandong University (September, 2018 - December, 2020). 


\section{Disclosure}

The authors declare that there are no conflicts of interest in this work.

\section{References}

1. Torre LA, Bray F, Siegel RL, Ferlay J, Lortettieulent J, Jemal A. Global cancer statistics, 2012. CA Cancer J Clin. 2015;65:87-108. doi:10.3322/caac. 21262

2. Kessler ER, Dan T, Flaig TW. Molecular Targeted Therapy of Bladder Cancer. Precision Molecular Pathology of Bladder Cancer [J]. 2018.

3. Liu X, Cheng X, Liu X, He L, Ji Z. Investigation of the urinary metabolic variations and the application in bladder cancer biomarker discovery. Int J Cancer. 2018;143(2). doi:10.1002/ijc.31323

4. Xie YZ, Yang F, Tan W, et al. The anti-cancer components of Ganoderma lucidum possesses cardiovascular protective effect by regulating circular RNA expression. Oncoscience. 2016;3:203-207. doi:10.18632/oncoscience.316

5. Zhu Q, Lu G, Luo Z, et al. CircRNA circ_0067934 promotes tumor growth and metastasis in hepatocellular carcinoma through regulation of miR-1324/FZD5/Wnt/3-catenin axis. Biochem Biophys Res Commun. 2018;497(2):626. doi:10.1016/j.bbrc.2018.02.119

6. Li R, Ke S, Meng F, et al. CiRS-7 promotes growth and metastasis of esophageal squamous cell carcinoma via regulation of miR-7/ HOXB13. Cell Death Dis. 2018;9:1-13.

7. Du WW, Yang W, Chen Y, et al. Foxo3 circular RNA promotes cardiac senescence by modulating multiple factors associated with stress and senescence responses. Eur Heart J. 2016;38:1402.
8. Segura MF, Hanniford D, Menendez S, et al. Aberrant miR-182 expression promotes melanoma metastasis by repressing FOXO3 and microphthalmia-associated transcription factor. Proc Natl Acad Sci U S A. 2009;106(6):1814-1819. doi:10.1073/pnas.0808263106

9. Ji H, Tian D, Zhang B, Zhang Y, Yan D, Wu S. Overexpression of miR-155 in clear-cell renal cell carcinoma and its oncogenic effect through targeting FOXO3a. Exp Ther Med. 2017;13(5):2286-2292. doi:10.3892/etm.2017.4263

10. Sisci D, Maris P, Grazia Cesario M, et al. The estrogen receptor $\alpha$ is the key regulator of the bifunctional role of FoxO3a transcription factor in breast cancer motility and invasiveness. Cell Cycle. 2013;12 (21):3405-3420. doi:10.4161/cc.26421

11. Lu W-Y. Roles of the circular RNA circ-Foxo3 in breast cancer progression. Cell Cycle. 2017;16(7):589-590. doi:10.1080/15384 101.2017.1278935

12. Du WW, Fang L, Yang W, et al. Induction of tumor apoptosis through a circular RNA enhancing Foxo3 activity. Cell Death Differ. 2017;24 (2):357-370

13. Sand M, Skrygan M, Georgas D, et al. Microarray analysis of microRNA expression in cutaneous squamous cell carcinoma. J Dermatol Sci. 2012;68(3):119-126

14. Mishra PJ, Bertino JR. MicroRNA polymorphisms: the future of pharmacogenomics, molecular epidemiology and individualized medicine. Pharmacogenomics. 2016;10:399-416. doi:10.2217/14622416.10.3.399

15. Cui QK, Liu WD, Zhu JX, Wang YH, Wang ZG. MicroRNA-184 promotes proliferation ability of glioma cells by regulating FOXO3. Asian Pac J Trop Med. 2014;7:776-779. doi:10.1016/S1995-7645(14) 60135-8

\section{Publish your work in this journal}

Cancer Management and Research is an international, peer-reviewed open access journal focusing on cancer research and the optimal use of preventative and integrated treatment interventions to achieve improved outcomes, enhanced survival and quality of life for the cancer patient.
The manuscript management system is completely online and includes a very quick and fair peer-review system, which is all easy to use. Visit http://www.dovepress.com/testimonials.php to read real quotes from published authors. 